\title{
Fatale Klarheit
}

\section{Dieter Birnbacher}

Online publiziert: 11 . November 2011

(C) Springer-Verlag 2011

Die Verlautbarungen der Bundesärztekammer zur Frage des ärztlich assistierten Suizids beschreiben seit den Richtlinien von 1993 die Bewegung der Echternacher Springprozession im Krebsgang: ein Schritt vor, zwei Schritte zurück. 1993 bezeichneten die Richtlinien für die ärztliche Sterbegleitung die Mitwirkung des Arztes bei der Selbsttötung eines Patienten als ,unärztlich“ - eine Wertung, die die Grundsätze der Bundesärztekammer von 1998 und 2004 in leicht abgewandelten Formulierungen übernahmen. Standesrechtliche Sanktionen, wie sie im Jahr 1988 von der Bayerischen Ärztekammer gegen Julius Hackethal zur Anwendung kamen, waren damit nicht mehr intendiert. Der Arzt, der einem Patienten Beihilfe zum Suizid leistete, musste bei Bekanntwerden jedoch mit einer Rufschädigung rechnen. Anfang 2011 kam es dann zu einer überraschenden Liberalisierung: In den Grundsätzen der Bundesärztekammer vom 21.1.2011 hieß es nur noch: „Die Mitwirkung des Arztes bei der Selbsttötung ist keine ärztliche Aufgabe“, eine an die Schweizer Richtlinien von 2005 angelehnte Formulierung, die, wenn man der dort implizit vorausgesetzten Lesart folgt, nicht mehr besagt, als dass kein Arzt verpflichtet ist, der Bitte eines Patienten nach Suizidbeihilfe nachzukommen. Die Berechtigung zur Suizidbeihilfe wird durch die Aussage aber ebensowenig in Frage gestellt wie durch die frühere Aussage, dass sie „dem ärztlichen Ethos widerspricht“. Auch für andere gesellschaftlich umstrittene Maßnahmen, etwa Schwangerschaftsabbrüche nach der Fristenregelung und chirurgische Eingriffe zu ausschließlich ästhetischen Zwecken ist zweifelhaft, ob sie dem ärztlichen Ethos entsprechen, ohne dass ihre Ausführung deshalb Ärzten umstandslos verwehrt ist. Mochten es die bisherigen Formulierungen in diesem Punkt an Eindeutigkeit fehlen lassen, ist mit dem jähen Schwenk der Bundesärztekammer zu einem rigiden Verbot nunmehr vollständige Klarheit hergestellt: Am 1.6.2011 beschloss der Bundesärztetag auf Anregung der Bundesärztekammer, in die Musterberufsordnung einzufügen, dass Ärzte „,keine Hilfe zur Selbsttötung leisten dürfen“. Rechtsverbindlich wird dieser Beschluss allerdings erst, wenn die geänderte Musterberufsordnung von den Bundesländern in das ärztliche Berufsrecht übernommen wird.

Prof. Dr. D. Birnbacher $(\bowtie)$

Heinrich-Heine-Universität Düsseldorf, Institut für Philosophie,

Universitätsstr. 1, 40225 Düsseldorf, Deutschland

E-Mail: dieter.birnbacher@uni-duesseldorf.de 
In der Schweiz - von der rechtlichen Situation Deutschland vergleichbar - hat die Richtlinienentwicklung eine genau umgekehrte Richtung genommen. Ausgehend von einer ähnlichen Formulierung wie der in den deutschen Grundsätzen relativieren die Richtlinien der Schweizerische Akademie der Medizinischen Wissenschaften von 2004 diese Aussage durch den Nachsatz: „Auf der anderen Seite ist die Achtung des Patientenwillens grundlegend für die Arzt-Patient-Beziehung. Diese Dilemmasituation erfordert eine persönliche Gewissensentscheidung des Arztes. Die Entscheidung, im Einzelfall Beihilfe zum Suizid zu leisten, ist als solche zu respektieren." Die Schweizerische Nationale Ethikkommission hat daraufhin den weiteren Schritt getan zu fordern, dass „,der wohl erwogene persönliche Entschluss zum Suizid [...] nicht an Regeln einer Institution, dem persönlichen Gewissensentscheid eines einzelnen Arztes oder einer einzelnen Betreuungsgruppe scheitern müsse“. Es soll vielmehr die Möglichkeit gewährt werden, ,,auf Wunsch einem anderen Arzt zugewiesen oder in eine andere Institution verlegt zu werden".

Die Wechselhaftigkeit der Äußerungen der deutschen Ärzteschaft ist ein Indiz für eine tiefgreifende Verunsicherung angesichts eines zunehmenden Meinungspluralismus innerhalb und außerhalb der Ärzteschaft. Der Versuch, die Unsicherheit mit der rigidest möglichen Lösung zu bewältigen, muss aber als ein klarer Rückschritt gewertet werden - als Versuch, eine antiquierte Rollenidentität wiederherzustellen. In der Realität hat sich das Aufgabenspektrum der Ärzte massiv gewandelt. Längst hat sich das Spektrum ärztlicher Tätigkeiten über die herkömmlichen „Ziele der Medizin“ hinaus in Bereiche hinein ausgedehnt, in denen es nicht mehr um Krankheit und ihre Heilung, sondern um die Bewältigung von Lebensproblemen geht, soweit sich diese mit den Mitteln der Medizin erleichtern lassen. Dazu gehören so unterschiedliche Maßnahmen wie Geburtenregelung, psychotherapeutische Beratung bei Partnerproblemen und Aufklärung und Hilfestellung bei der Erstellung von Patientenverfügungen. Ziel ist jedesmal nicht die Heilung, Linderung oder Verhinderung von Krankheiten und ihrer Symptome, sondern eine durch ärztliche Hilfe ermöglichte oder erleichterte selbstbestimmte Lebensführung. Medizinische Hilfe wird nachgesucht nicht, um zu leben, sondern um gemäß den eigenen Wertvorstellungen zu leben. Dazu gehört auch ein selbstbestimmtes Sterben. Gerade an den Lebensgrenzen bedarf es ärztlicher Hilfe, wenn Selbstbestimmung nicht nur proklamiert, sondern auch real gelebt werden soll. Wie bei der selbstbestimmten Familienplanung verbürgen nur Ärzte die erforderliche Sachkenntnis und die Beachtung der einschlägigen Sorgfaltskriterien. Nur Ärzte können kompetent beurteilen, wie weit der Hilferuf eines Patienten Ausdruck einer behandelbaren Depression oder anderen psychischen Störung ist und wie weit er auf eine reale und dauerhafte Notlage zurückgeht, in der der Patient nach seinen Maßstäben unerträglichem Leiden ausgesetzt ist und für sich dauerhaft keine positive Lebensperspektive mehr sieht. Dieses Urteil und die Hilfe beim Suizid in die Hände von Nicht-Ärzten zu legen, ist in der Mehrzahl der Fälle belastender und risikoreicher.

Der Beschluss ist nicht nur rückwärtsgewandt, er ist auch unter moralischen Gesichtspunkten bedenklich. Er nimmt schwerkranken Patienten, die auf eine selbstbestimmte Beendigung ihres Lebens hoffen, die Aussicht, dafür wenn nicht auf ihren Hausarzt, so doch zumindest auf einen anderen Arzt ihres Vertrauens zählen zu können. Bei allen Fortschritten der Palliativmedizin ist weiterhin davon auszugehen, dass die Lage vieler Todkranker verzweifelt ist und Todkranke sich einen früheren als den ,natürlich“ eintretenden Tod intensiv wünschen. Dieser Wunsch ist auch bei vielen Patienten ausgeprägt, die den Suizid für sich und andere aufgrund ihrer religiösen oder weltanschaulichen Orientierung in gesunden Tagen kategorisch abgelehnt haben. Diesen Patienten wird eine der für sie am wenigsten ängstigenden Optionen versperrt. 
Bedenklich ist der Beschluss auch insofern, als er tendenziell in ein grundlegendes Freiheitsrecht eingreift. Die Legitimität der internen ärztlichen Normsetzung muss ihre Grenze da finden, wo Grundrechte des Bürgers berührt sind. Auch wenn das Recht auf Suizid (noch) nicht juridisch anerkannt ist, gilt es doch spätestens seit der Aufklärung ethisch als Teil des Freiheitsrechts. Ein Freiheitsrecht ist aber stets nur so viel wert, wie es nicht nur in abstracto garantiert ist, sondern eine angemessene Chance besteht, es konkret in Anspruch zu nehmen.

Die Argumente auf der Gegenseite sind denkbar schwach: Dafür, dass die Bereitschaft von Ärzten, einem schwerkranken Patienten bei einem Suizid zu helfen, das Vertrauen der Patienten in den ärztlichen Stand untergräbt, gibt es keinen Beleg. In einer Forsa-Umfrage im November 2003 stimmten $84 \%$ der deutschen Bevölkerung der Aussage zu: „Wenn mein Hausarzt einem unheilbar kranken Patienten bei der Selbsttötung hilft, würde ich das Vertrauen zu ihm nicht verlieren." Schwach ist auch das von vielen zu hörende Dammbruchargument: Wenn Ärzte dem Tod statt dem Leben dienen dürfen, besteht die Gefahr, dass Suizidwünsche wahllos erfüllt werden. Dass in dieser Hinsicht ein Risiko besteht, ist schwer zu leugnen. Aber statt ein gnadenloses Verbot zu erlassen, sollte es Anlass sein, über mögliche Kontrollmöglichkeiten nachzudenken. Auch der Umgang mit Opiaten birgt erhebliche Risiken. Dennoch kommt keiner auf den Gedanken, sie deshalb und ungeachtet des Guten, das sie tun, zu verbieten. 